\title{
Solar-Heliospheric Physics
}

\section{Ruffolo*a}

${ }^{a}$ Department of Physics, Faculty of Science, Mahidol University, Bangkok 10400, Thailand.

\section{E-mail: david.rufemahidol.ac.th.}

This rapporteur paper summarizes the 99 presentations on solar-heliospheric physics at the 35th International Cosmic Ray Conference. These spanned a dizzying array of physical phenomena, especially time-dependent phenomena associated with solar storms, the 27-day solar rotation, the $\sim 11$-year sunspot cycle, and the $\sim 22$-year solar magnetic cycle. There was also work on composition-dependent effects and cosmic ray anisotropy involving transport effects. Topics included ground- and space-based instrumentation, solar energetic particles from solar storms (ranging from keV-range seed particles to $\mathrm{GeV}$-range accelerated particles), interplanetary transport of energetic particles, Earth's radiation environment (including space weather and other effects of cosmic rays at Earth), the sun shadow in TeV-range Galactic cosmic rays (GCRs), and solar modulation of GCRs with the sunspot cycle and solar magnetic cycle.

35th International Cosmic Ray Conference

12-20 July, 2017

Bexco, Busan, Korea

*Speaker. 


\section{Introduction}

It has been a privilege to be invited to summarize the presentations in the solar-heliospheric (SH) sessions of the 35th International Cosmic Ray Conference. The presentations have been categorized as shown in Table 1. Each section and subsection of this report lists in parentheses the number of presentations on that range of topics. Here I discuss all 99 contributed presentations that were actually delivered, either orally or by putting up a poster. The presenters are to be commended for their wonderful variety and scientific depth, and also for the congenial atmosphere and wideranging discussion of these topics.

Table 1: Number of presentations in various categories of solar-heliospheric physics.

\begin{tabular}{lr}
\hline \hline Category & Number \\
\hline Instrumentation & 9 \\
Solar Energetic Particles & 27 \\
Interplanetary Transport & 9 \\
Radiation Environment at Earth & 19 \\
Sun Shadow in TeV-Range Cosmic Rays & 3 \\
Solar Modulation of Galactic Cosmic Rays & 32 \\
\hline
\end{tabular}

Solar-heliospheric topics of cosmic ray physics concern particles accelerated within the heliosphere, especially solar energetic particles (SEPs) accelerated by solar storms and their space weather effects at Earth, and also time variations of Galactic cosmic rays (GCRs) due to the variable solar wind and solar storms. To stress the important relations between these phenomena, all the relevant particle populations will be collectively referred to as "cosmic rays." Naturally it is also necessary to consider the transport of these cosmic ray particles as they traverse turbulent magnetic fields in the heliosphere to the point of observation. These particle populations and transport processes are illustrated in Figure 1. The energies of interest range from the keV range (e.g., seed particles for acceleration at interplanetary shocks) to the $\mathrm{TeV}$ range (e.g., regarding time variations of the sun shadow). Observations of interest to the presentations at this conference were mostly from spaceborne instruments (for direct detection of particles in the $\mathrm{keV}$ to $\mathrm{GeV}$ ranges, and for in situ exploration of the inner heliosphere as well as the outer heliosphere and local interstellar plasma) or ground-based detectors of atmospheric secondary particles (for indirect detection of relativistic ions in the $\mathrm{GeV}$ to $\mathrm{TeV}$ ranges). These observations also concern the species and composition dependence of those particle populations. Socially, the solar-heliospheric sessions were distinct from other types of cosmic ray research in that most presentations were based on research by small groups, even among observational reports.

Finally, note that time variations were a universal theme of the solar-heliospheric presentations. Sources of time variation include solar storms, the 27-day solar rotation (especially as it affects the solar wind speed at a given point in the heliosphere), the $\sim 11$-year sunspot cycle, and the $\sim 22$-year solar magnetic cycle. Furthermore, for observations from Earth, an orbiting satellite, or a rotating spacecraft, the anisotropy of cosmic rays in space can be manifest in time variations, e.g., "diurnal variations" with a 1-day period due to Earth's rotation. 
Figure 1: Illustration of some of the solarheliospheric topics of cosmic ray physics, including studies of solar energetic particles accelerated by solar storms, time variations of Galactic cosmic rays due to the variable solar wind and solar storms, and the underlying transport processes.

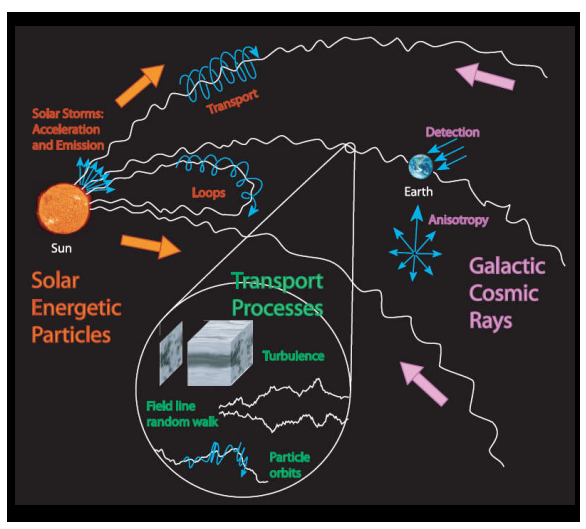

\section{Instrumentation (9)}

There was a report on the capabilities and performance of the High-Energy Energetic-Particles Instrument for the Parker Solar Probe Mission [103] (Figure 2). This exciting mission will measure energetic particles as close as $0.04 \mathrm{AU}$ from the Sun!

Figure 2: EPI-Hi silicon detector suite for the Parker Solar Probe mission to approach the Sun, mounted in a handling fixture prior to delivery to the spacecraft in May 2017. For scale: the LET1 window has a diameter of $4.2 \mathrm{~cm}$ [103].

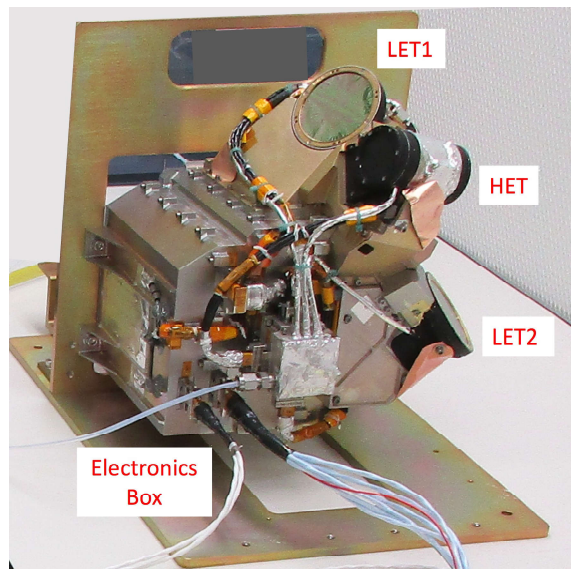

There were many reports concerning measurements by neutron monitors (NMs). These are ground-based detectors of atmospheric secondary particles that are most sensitive to secondary neutrons with energies between $10 \mathrm{MeV}$ and $1 \mathrm{GeV}$, which are mainly produced by primary cosmic rays in the GeV range. The standard NM64 detector design is illustrated in Figure 3. The energy response to primary cosmic rays is strongly affected by Earth's magnetic field, which serves as a magnetic spectrometer. At each location on Earth, there is a geomagnetic cutoff (threshold) rigidity (i.e., momentum per charge, in units of $\mathrm{GV}$ ) for ground-based detection of cosmic rays. The geomagnetic cutoff varies from $\sim 1 \mathrm{GV}$ in polar regions to $17 \mathrm{GV}$ in parts of South and Southeast Asia. For atmospheric secondary particles to be detected at ground level, the particle rigidity should also exceed an atmospheric cutoff of $\sim 1 \mathrm{GV}$. Because the cutoff depends on the detector location, NMs have been constructed in many locations around the world (see Figure 4). Many NMs are located at high mountain altitudes, where the count rate can be several times higher than at sea level. 
Figure 3: Top: View inside the Princess Sirindhorn Neutron Monitor at Doi Inthanon, Thailand, showing the 18-tube NM64 and 3 bare counters. Bottom: Components of the NM (to scale). $\mathrm{Pb}$ : Lead producer "rings with wings," in which a cosmic-raygenerated atmospheric secondary particle, typically a neutron of $10 \mathrm{MeV}$ to $10 \mathrm{GeV}$, can disrupt a lead nucleus to produce several low-energy neutrons. PE: Polyethylene, through which high-energy neutrons typically pass without interacting, but lowenergy neutrons are efficiently moderated toward the thermal energy range. The 3-inch $\mathrm{PE}$ reflector surrounding the monitor serves to block low-energy neutrons from the environment while trapping most neutrons produced by interactions in the lead. PC: ${ }^{10} \mathrm{BF}_{3}$ gas proportional counter tube (viewed in cross-section) in which neutrons (especially those at low energy) interact with ${ }^{10} \mathrm{~B}$ and the reaction products produce a strong, characteristic electronic signal. Wood: Wooden supports for the lead rings [84].
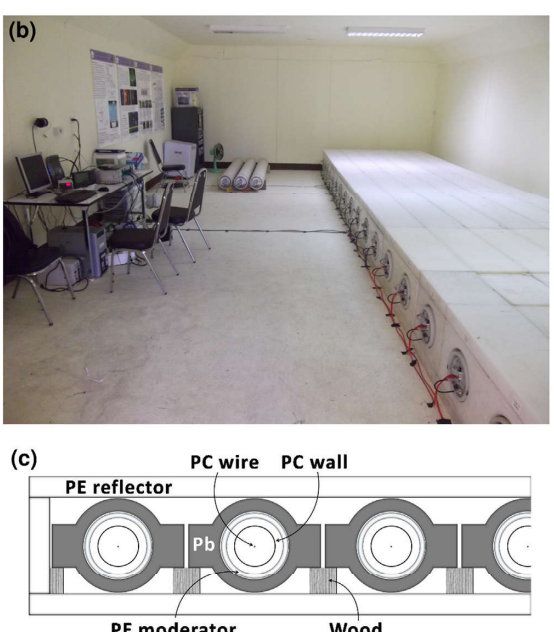

Figure 4: Geographic distribution of active NM stations, along with contours of constant geomagnetic cutoff rigidity [75].

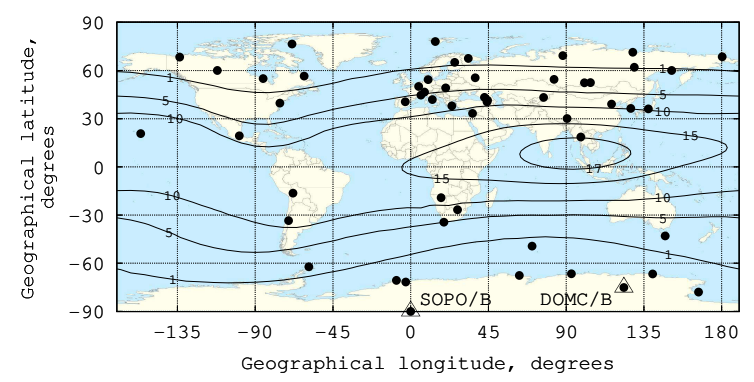

There were reports of new electronics for NMs [43] and the results from specialized electronics that record histograms of time delays between successive neutrons [86]. The latter report showed measurements of the "leader fraction" (fraction of neutron counts that did not follow another temporally associated neutron count from the same primary cosmic ray, or inverse multiplicity) measured on different counter tubes at Doi Inthanon, Thailand, as a function of counter separation distance. At low tube separation, a low leader fraction (high multiplicity) is associated with the spread of neutrons from a common interaction point. However, Figure 5 shows that as the separation increases, the leader fraction saturates at a value lower than one, which indicates the detection of neutrons from multiple atmospheric secondaries in different parts of the detector.

Other work concerned the design and construction of mini-NMs [41], which are smaller and cheaper NMs (with lower count rates). A mini-NM has been deployed at $4600 \mathrm{~m}$ altitude at Sierra Negra, Mexico [45]. Another innovative ground-based detector is the SciBar Cosmic Ray Telescope (SciCRT) [6, 7,21], with 15,000 scintillator bars and dimensions of $1.7 \times 3 \times 3 \mathrm{~m}^{3}$, which is also deployed at Sierra Negra (Figure 6). The purpose is to detect neutrons and muons. Finally, another report described the Solar Influence on Decay Rate (SIDR) experiment, designed to monitor the decay rate of ${ }^{90} \mathrm{Sr}[10]$. 
Figure 5: Cross-counter leader fraction $L_{\Delta}$ as a function of counter separation $\Delta$ in the neutron monitor at Doi Inthanon, Thailand. The value less than one at large separations indicates the detection of multiple atmospheric secondaries from the same primary cosmic ray [86].

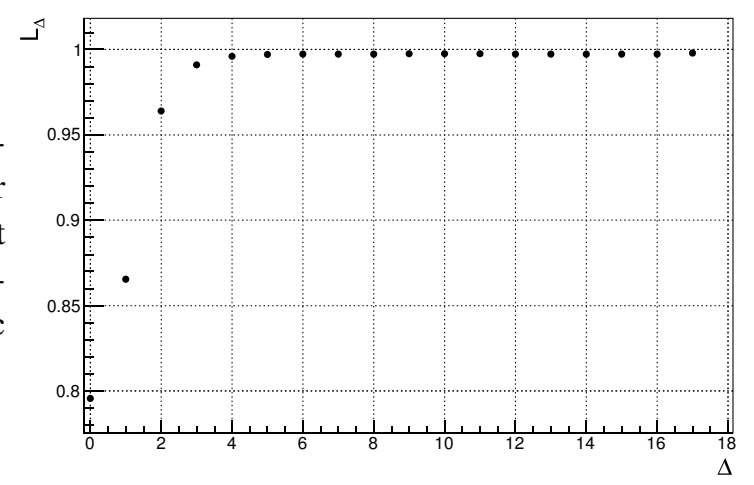

Figure 6: A picture of SciCRT installed on Sierra Negra volcano [7].

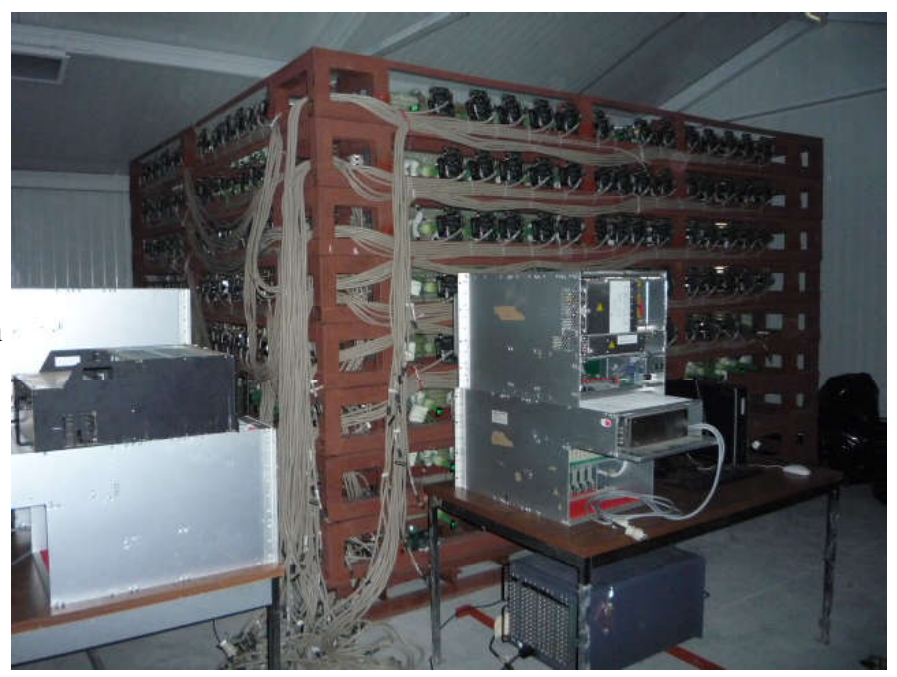

\section{Solar Energetic Particles (27)}

Solar energetic particles (SEPs) are accelerated in association with solar storms. Solar storms are occasional explosions at the surface of the Sun, almost always at sunspot groups (active regions), involving rapid conversion of magnetic energy, through magnetic reconnection, to heat energy and/or kinetic energy. A solar flare is a heating event, often identified in terms of thermal $\mathrm{X}$-ray emission, and a coronal mass ejection (CME) represents conversion of magnetic energy to kinetic energy of a large volume of tenuous gas. Flares can accelerate ambient particles by stochastic (turbulent) acceleration over rapid time scales, with ${ }^{3} \mathrm{He}$ and heave element enhancements. A fast CME will drive an interplanetary shock through the solar wind plasma, and ambient seed particles can experience diffusive shock acceleration. A highly energetic solar storm will usually have both a flare and a CME, in which case prior work has shown that escaping energetic ions are predominantly from the shock.

\subsection{Ground Level Enhancements and Other Extreme Solar Storms (9)}

There was extensive discussion of ground level enhancements (GLE) and other extreme solar storms. Ground-based detectors always measure GCRs, but on occasion a solar storm produces 
such a huge flux of relativistic solar particles that the flux is noticeable above the GCR "background." A related phenomenon is a temporary Forbush decrease (FD) in GCR upon the arrival of the CME-driven shock, sheath region, and/or CME at Earth. As an illustration, Figure 7 shows NM rates for a sequence of three major solar storms in 1989, each of which was associated with a GLE just a few minutes after the solar flare (labeled "F") and a FD up to two days later near the time of shock arrival at Earth (labeled "S").

Figure 7: Count rates of NMs at McMurdo (Antarctica) and Thule (Greenland) in 1989 October, which are mostly due to GeV-range Galactic cosmic rays (GCRs). There was also a sequence of 3 ground level enhancements (GLEs) representing relativistic ions near the time of a major solar flare ("F"), each followed by a Forbush decrease (FD) in Galactic cosmic rays near the time of arrival at Earth of an interplanetary shock ("S"). Because these two neutron monitors view different directions in the sky, the major difference between the two traces for the October 22 GLE (inset) indicates an unusually strong anisotropy in relativistic solar protons. Adapted from [85].

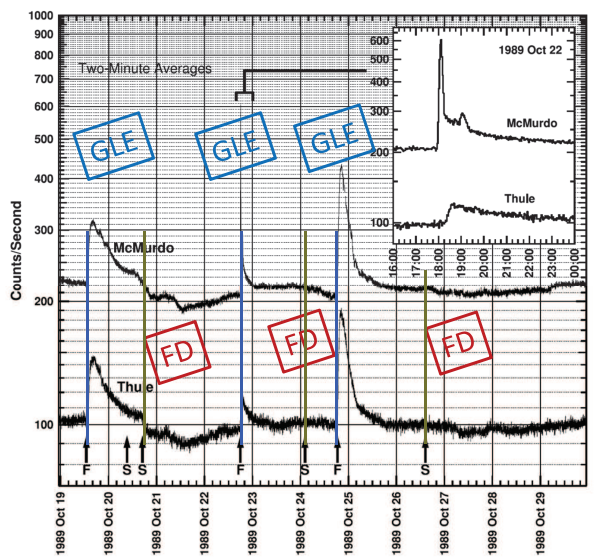

While the previous Solar Cycle 23 had 15 GLEs, including one with a 55-fold increase in radiation at the South Pole (on 2005 Jan 20), the present Solar Cycle 24, up to the time of the conference, had only 3 weak events detected by multiple ground-based detectors [32]. Furthermore, one team proposed that one or more of the small events should not be called GLEs [75]. The classic definition of a GLE is an enhancement at two or more NMs, but these authors view that high-altitude polar NMs are particularly sensitive to solar vs. Galactic cosmic rays and have an "unfair" advantage compared with other NMs. It is only recently that multiple such detectors have been in operation (the NMs at South Pole and Dome C, and IceTop at South Pole). In the interest of standardization, they propose that an event only observed by multiple high-altitude detectors should instead be termed a "sub-GLE."

Indeed, it would be useful to have a term for extreme solar radiation events based on SEP properties, not based on a type of detector [17]. For example, the STEREO spacecraft observed some very large SEP events that were not directed to Earth. It was proposed that important properties of extreme events are a broken power-law spectrum of SEPs with a hard upper power law (see also [95]) and heavy element enhancements. Magnetic connectivity to two CME-driven shocks may also play a role.

A worst-case scenario of space weather effects was considered by [94] for the strongest known SEP event, in 775 A.D. The pulse shape of GLEs was considered by [41]. The origin of two extreme solar particle events was described by [37]. Other work outlined a procedure for analyzing data on GLEs from the global NM network [57]. They use data from NMs at varying geomagnetic cutoff, calculate asymptotic look directions using the Tsyganenko model of the (disturbed) geomagnetic field, fit parameters for the spectrum and pitch angle distribution, their NM yield functions, and special considerations for "sub-GLEs." Finally, there was work to analyze paper traces for the giant GLE of 1956 Feb 23 from older ionization chambers in order to compare with NM data [51]. 
Figure 8: Integrated fluence of $>10$ $\mathrm{MeV}$ protons versus the day of the cycle for Solar Cycles 22, 23, and 24. [54].

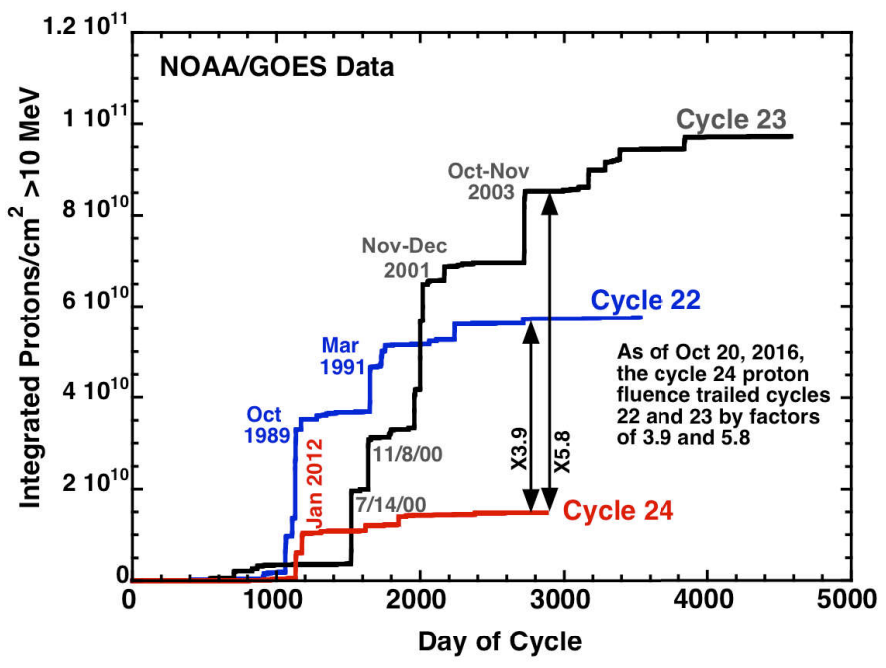

\subsection{Spectra (5)}

The issue of why the present Solar Cycle 24 produced so few strong SEP events, with a much lower SEP fluence than the two preceding solar cycles (see Figure 8), was addressed by [54]. During strong events, SEP spectra have broken power laws (changing at $E_{\text {break }}$ ) and a high-energy rollover (at $\sim E_{\max }$ ). Thus the overall fluence during a solar cycle depends on $E_{\text {break }}$ and $E_{\max }$. The authors note that CME-driven shocks preferentially accelerate ambient suprathermal (up to keVrange) ions, not thermal solar wind ions, avoiding the "injection problem" for shock acceleration of thermal particles. One proximate cause of the lower SEP fluence in Solar Cycle 24 is the lower suprathermal flux, which was $\sim 4$ times weaker than that in the previous solar cycle (see also [31]). What are the ultimate causes for that? [54] identified that the present cycle had fewer fast CMEs and a weaker magnetic field. Such effects are amplified by the nonlinear nature of the shock acceleration process: with lower suprathermal proton injection, there was weaker turbulence generation. The power-law break energy $E_{\text {break }}$ indicates the energy at which particles are no longer trapped at the shock, and with weaker turbulence then $E_{\text {break }}$ was lower. Also, $E_{\max }$ was somewhat lower because of the weaker magnetic field, though this is considered to be of only secondary importance. As a result of the reduced $E_{\text {break }}$ and $E_{\max }$, the SEP fluence was greatly reduced, a conclusion that was supported by numerical simulations using the iPATH program.

Another report addressed the question of why there is a spectral break in strong SEP events by examining $E_{\text {break }}$ for different elements and its dependence on the charge-to-mass ratio $Q / M$ [18]. In particular, for all SEP events examined they found a dependence $E_{\text {break }} \propto(Q / M)^{\alpha}$ for $\alpha$ between 0.2 and 2, with $\alpha \geq 1.4$ for extreme SEP events. This is considered to be consistent with acceleration-dominated models for the break and inconsistent with transport-dominated models. The enrichments in ${ }^{3} \mathrm{He}$ and heavy elements (the latter is indicated by high $\alpha$ ) for extreme SEP events is interpreted in terms of enriched turbulent power enabling CME shocks near the Sun to acceleration flare-rich material more efficiently than ambient coronal material.

In addition, there was a report of SEP spectra as measured by the PAMELA satellite instrument [53]. There were also simulations of enhanced acceleration and spectra for two CMEs (a prior CME and a new CME) [99]. 


\subsection{Correlations (2)}

There was a report on the correlation between the peak SEP flux with the reconstructed 3D CME speed and the relative longitude [67]. Other work found that the peak SEP proton flux is sometimes better correlated with the flare X-ray intensity than the CME speed [47].

\subsection{Solar Gamma Rays and Neutrons (4)}

In one report, signals at high-mountain solar neutron telescopes were interpreted in terms of solar gamma rays penetrating Earth's atmosphere during events on 2011 Mar 7 and 2011 Sep 25 [65]. This interpretation was disputed by another report [12]. There was work on the detection efficiency of the solar neutron telescopes located at high altitudes [30], and the sensitivity of SciCRT (see Section 2) to solar neutrons [87].

\subsection{Solar Energetic Particles at the Sun (3)}

There were three reports concerning SEP interactions at the Sun with regard to neutral emissions that could be observed at Earth. There were simulations of the angular distributions of the resulting solar gamma rays and neutrons [35]. There was also work on how gamma ray and possible neutron observations at Earth orbit can be used to infer the interacting solar proton flux [36]. It was determined that interacting solar protons have only a weak contribution to white-light solar flares [101].

\subsection{Other (4)}

As examples of the great variety of topics in solar-heliospheric cosmic-ray physics, there were reports on other aspects of SEP events. The high energy ionic charge states $Q$ during SEP events from 2005 to 2016 were re-analyzed using data from the ACE and STEREO spacecraft [44]. This work makes use of techniques to infer $Q$ from time-intensity profiles, which are particularly useful when direct charge state measurements or measurements based on the geomagnetic cutoff were not available. There was a report on peculiar anisotropy oscillations during the $2012 \mathrm{Jul} 23$ SEP event [48]. There was a study of how cosmogenic isotopics in lunar samples relate to the SEP flux [74]. As previously noted, there was a study of SEP and suprathermal particle variations of Solar Cycle 24, using ACE/ULEIS data [31].

\section{Interplanetary Transport (9)}

\subsection{Fundamentals (5)}

Several presentations were devoted to the fundamental physics of cosmic ray transport in the heliosphere, i.e., through the magnetic turbulence of the solar wind. One study examined the geometry of solar wind magnetic turbulence at Earth orbit [14]. Other work examined the reduction of cosmic-ray drift coefficients in the presence of turbulence, throughout the heliosphere [19]. Toroidal models of force-free magnetic fields were also considered [70], as well as a stochastic solution of Parker's transport equation in a fully five-dimensional space [102] and solar proton transport to Earth [13]. 


\subsection{Lateral Transport of Solar Energetic Particles (2)}

While SEP transport is often considered to be dominated by "parallel transport" along the Parker spiral magnetic field from the Sun to Earth and beyond, there are also interesting transport effects in the lateral directions (i.e., in heliolatitude and heliolongitude), which require some motion perpendicular to the large-scale field. [90] stressed the transport of solar energetic electrons, treating the perpendicular transport as diffusive (see Figure 9). In contrast, [93] stressed that the initial lateral transport of SEPs is not diffusive (see Figure 10), because even a tiny diffusion coefficient would wash out gradients in particle density, in conflict with observations by the ACE spacecraft of sharp dropouts (decreases and increases) in the density of SEPs from impulsive solar events. This work also expressed predictions regarding SEP time profiles near the Sun as will soon be observed by the upcoming Parker Solar Probe and Solar Orbiter missions.

Figure 9: Density of $\sim 100 \mathrm{keV}$ solar energetic electrons from an injection region $0.05 \mathrm{AU}$ from the Sun after $5 \mathrm{~h}$, as modeled using perpendicular diffusion [90].
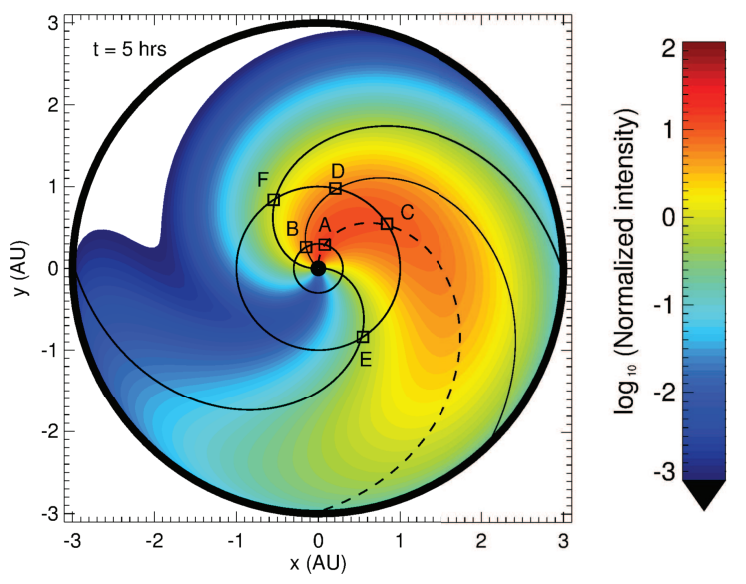

Figure 10: Trajectories of 50,000 magnetic field lines in heliolongitude and heliolatitude as a function of radius $r$ from the Sun, starting at random initial locations at $r=0.1$ AU within a circle of radius $2.5^{\circ}$, for the 2D MHD + slab magnetic fluctuation model. From full-orbit calculations, particle trajectories are found to initially follow these field line trajectories. This dropout pattern is consistent with prior observations by the ACE spacecraft [93].
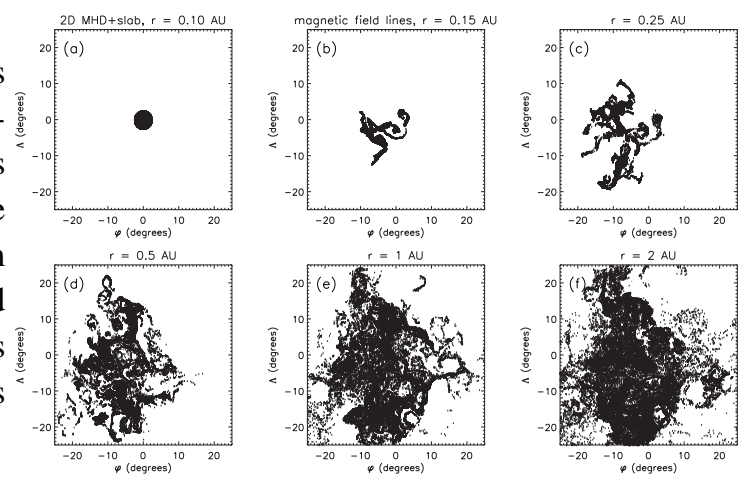

\subsection{Shock Effects (2)}

There was also work on upstream wave excitation and particle scattering at Earth's foreshock, which results from interaction of the solar wind with Earth's magnetosphere [66], and high-energy cosmic ray modulation associated with interplanetary shocks [11]. 


\section{Radiation Environment at Earth (19)}

\subsection{Space Weather Effects of Solar Storms (12)}

There were numerous presentations concerning the space weather effects of solar storms. One effect that is specific to relativistic solar particles is atmospheric ionization at aircraft altitudes or below. Monte Carlo simulations were shown by [58] for atmospheric ionization due to two GLEs in the previous Solar Cycle 23, on 2000 Jul 14 and 2006 Dec 13. Interestingly, in each case the average ionization over the week after the event at $8 \mathrm{~km}$ altitude is calculated to be negative because the Forbush decrease in Galactic cosmic rays offset the increase due to relativistic solar particles.

There were also simulations of atmospheric ionization due to the largest recorded GLE on 1956 Feb 23 [96], and due to high energy proton or electron precipitation [55, 56]. There was an interesting report on the effect of Forbush decreases on the latent atmospheric energy [92]. Atmospheric ionization affects atmospheric pressure, so Forbush decreases cause observed pressure changes of 1 to 6 mbar in polar to mid-latitudes.

There was a study of spatial and energy distributions of high energy electron bursts precipitating from the inner radiation belt [1]. The temperature of the mesosphere at Yakutsk, Russia was measured during 1999-2013 and the authors examined the correlation with the solar cycle and geomagnetic storms $[4,5]$. Long-term variations in the natural thermal neutron flux were measured by PRISMA-YBJ at Yangbajing, China at an altitude of $4300 \mathrm{~m}$ [88]. It was reported that cosmic ray anisotropy changes before and during geomagnetic storms, which could provide a way to provide advance warning before storm onset [27]. Single event upset rates in electronics were calculated based on the measured SEP flux [79]. There was a study of the delay time of strong geomagnetic storms from CMEs at the Sun during 1999-2010 [83].

\subsection{Solar Cycle Effects (2)}

New types of possible solar cycle effects were proposed. There was an application of a coupled harmonic oscillator model to solar activity and El Niño phenomena [64]. Characteristics of global shallow-source seismicities were associated with solar activities in different time scales [105].

\subsection{Cosmogenic Nuclides (3)}

There are important historical records of the cosmogenic isotopes ${ }^{10} \mathrm{Be}$ in ice and ${ }^{14} \mathrm{C}$ in trees, which can tell us about cosmic rays at Earth in the past, e.g., for the largest known SEP event in 775 A.D. The production of the cosmogenic isotopes ${ }^{7} \mathrm{Be},{ }^{10} \mathrm{Be},{ }^{14} \mathrm{C},{ }^{22} \mathrm{Na}$, and ${ }^{36} \mathrm{Cl}$ in the atmosphere has been calculated by [73]. The issue of how solar activity caused peak anomalies of ${ }^{14} \mathrm{C}$ in 775 A.D. was considered by [67]. There was also a report of modern measurements of ${ }^{7} \mathrm{Be}$ at Bangkok, Thailand and modeling to study the observed seasonal variation [91]. The variation can be qualitatively accounted for using atmospheric wind trajectories from the HYSPLIT program, which come from different directions during different seasons, and using the location-dependent nuclide production calculated by EXPACS. However, there is also a need to account for scavenging of aerosols by rain. 
Figure 11: Deposition flux of ${ }^{10} \mathrm{Be}$ during last decades. The blue curve corresponds to the measurements of ${ }^{10} \mathrm{Be}$ in the NGRIP ice core in Greenland, and the red one represents the results of the production model presented here. The data are smoothed [73].

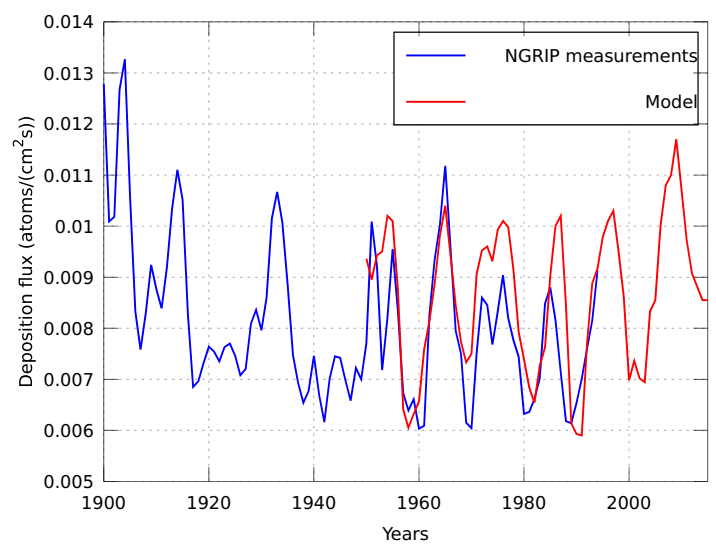

\subsection{Radiation from Thunderstorms (2)}

Our final topic on the radiation environment at Earth concerns a natural terrestrial source: lightning. The charge ratio of low energy muons was investigated using data during thunderstorms by [49]. Increases in scaler rates of water Čerenkov tanks in the HAWC detector were found during thunderstorms [46] and were attributed to relativistic runaway electron acceleration (not to muons).

Figure 12: The yearly deficit ratio of Sun shadow as a function of the median rigidity [16].

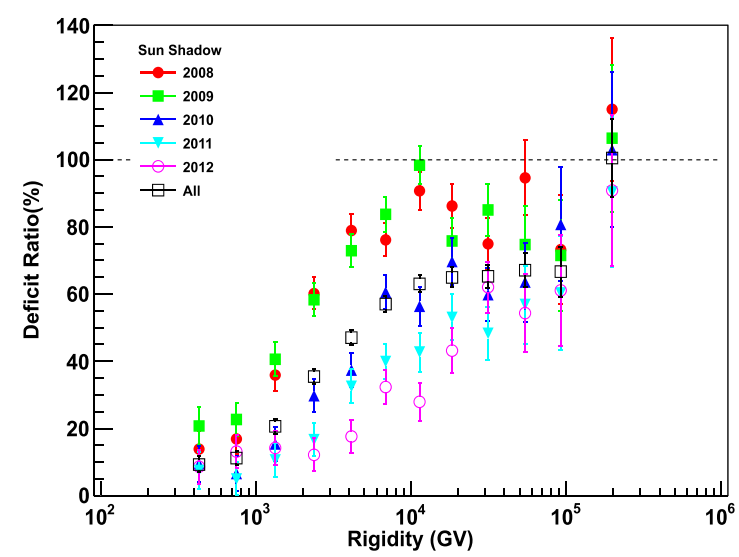

\section{Sun Shadow in TeV-Range Cosmic Rays (3)}

The sun shadow is a phenomenon where solar activity affects cosmic rays of even higher energy, up to $\sim 100 \mathrm{TeV}$. This meeting witnessed good progress on understanding the sun shadow and its dependence on the solar activity cycle. [3] reported on behalf of the Tibet AS $\gamma$ Collaboration that the sun shadow was much less clear during solar maximum. According to this collaboration [2], Monte Carlo simulations show that this effect of solar activity can mostly be attributed to the interplanetary magnetic field and partly due to the solar coronal field, and they even provided convincing evidence that interplanetary coronal mass ejections play a role. Details of the rigidity (energy) dependence of the sun shadow on the solar cycle variation over 2008-2012 as measured by ARGO-YBJ were presented by [16]. The deficit ratio, representing how strong the sun shadow was 
compared with the value expected from the geometric size of the Sun and the detector resolution, was about $20 \%$ or less at $\sim 0.5 \mathrm{TeV}$ during the entire time studied, implying that the sun shadow was greatly weakened by the processes listed above. However, at $\sim 4 \mathrm{TeV}$ the deficit ratio varied systematically from $\approx 80 \%$ at solar minimum (2008) to $\approx 15 \%$ near solar maximum (2012). Finally the deficit ratio was consistent with $100 \%$, with no reduction from the expected value, at $200 \mathrm{TeV}$ for all years studied. This rigidity dependence was qualitatively consistent with effects of either the interplanetary magnetic field or the solar coronal magnetic field.

\section{Solar Modulation of Galactic Cosmic Rays (32)}

The final category of presentations concerned the solar modulation of GCRs, and this category had the largest number of presentations. While the term "solar modulation" most commonly refers to effects of the $\sim 11$-year sunspot cycle and the $\sim 22$-year solar magnetic cycle, in this category we also include other studies of temporal variations in the GCR distribution, including changes in the GCR flux and anisotropy. The temporal variations are due to solar and heliospheric effects while the GCRs propagate from outside the heliosphere to Earth, including effects of the interplanetary magnetic field (IMF), magnetic turbulence, and solar wind speed [50]. While the longer-term variations provide remote sensing of large-scale heliospheric conditions, shorter-term variations are due to more local effects, such as 27-day variations in GCRs due the solar rotation and associated changes in the solar wind passing the detector's location, as well as temporary Forbush decreases in the GCR flux due to solar storms or high speed solar wind streams.

Figure 13: Long-term solar modulation of the Galactic cosmic ray flux as measured by the neutron monitor at Hermanus, South Africa, along with two proxies of solar activity. There is a strong variation in GCR flux with the $\sim 11$-year sunspot cycle. Also, near every sunspot maximum, there is a solar magnetic polarity reversal, marking a transition between " $q A-$ " and " $q A+"$ modulation. As a result, solar modulation also exhibits a variation with the $\sim 22$-year solar magnetic cycle (modified from presentation by [78]).

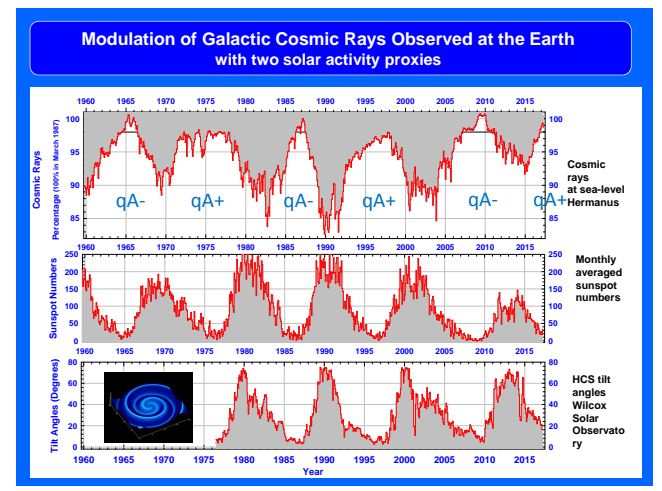

\subsection{Flux Variations (4)}

Figure 13 summarizes the long-term solar modulation of the GCR flux, based on data from the NM at Hermanus, South Africa, the currently active NM with the longest running dataset (since 1957). The most noticeable feature is the inverse association of the GCR flux with solar activity, e.g., with the sunspot number. Near the maximum of each $\sim 11$-year sunspot cycle, there is a magnetic polarity reversal in the Sun's polar regions, in which the preponderance of magnetic fields in the northern hemisphere reverses from outgoing (termed $A>0)$ to ingoing $(A<0)$ or viceversa. The preponderance of magnetic fields in the southern hemisphere is opposite to that in the North (except when the reversals occur at somewhat different times). Thus the Sun has a magnetic 
cycle of $\sim 22$ years. Because the cosmic rays of interest are charged, their transport through the heliosphere depends on the product $q A$. When we consider NM data as in Figure 13, the cosmic rays of interest are positive ions, so $q A$ has the same sign as $A$, and the $A>0$ periods exhibit "qA+" modulation conditions and $A<0$ periods exhibit "qA-" conditions.

With this in mind, the Figure shows GCR modulation not only with the $\sim 11$-year sunspot cycle but also with the $\sim 22$-year solar magnetic cycle. For example, the time profile for qAperiods is more sharply peaked and that for $\mathrm{qA}+$ periods is flatter. There are two basic reasons for these changes: polarity reversals involve changes in particle drifts and diffusion. The drift motion reverses, and particles enter the inner heliosphere either along the heliospheric current sheet (HCS, for $q A<0$ ) or along the heliospheric poles (for $q A>0$ ) [34]. In addition, diffusion coefficients change with changing solar magnetic polarity (as observed from diurnal anisotropy [15]), possibly due to helicity effects. The diffusion coefficients also depend on the magnetic field strength.

In this conference, [25] presented calculations of an effective energy of ground-based detectors for solar moduation. The 27-day variations due to solar rotation were shown to have an amplitude that varies with the 11-year and 22-year cycles [24]. There was a report of a possible periodicity with a period of 3-4 solar rotations, which could be attributed to differential rotation of the Sun [23]. Diffusion of cosmic rays in the heliosphere was discussed in the context of ground-based muon observations by GRAPES-3 [8].

\subsection{Spectral Variations (2)}

With regard to GCR spectral variations over a solar cycle, [50] reported a distinct pattern of solar modulation at high cutoff rigidity (at Doi Inthanon, Thailand) compared with that at low cutoff (at McMurdo, Antarctica). At solar maximum conditions (or specifically, when the HCS tilt angle was high), the modulation at high cutoff rigidity tracked the magnetic field intensity. This is interpreted to indicate that diffusion was short-circuiting the drift along the HCS for high-rigidity ions during times of high tilt angle.

There was also a presentation about measuring spectral variations at a single NM station, so as to avoid the systematic uncertainty of comparing rates between two stations [86]. Their method uses distributions of the time delay between successive neutron counts on a single counter tube, which were recorded hourly over a solar modulation cycle (2007-2017) at Doi Inthanon, Thailand, which has the world's highest cutoff rigidity for a fixed NM station $\left(P_{c} \approx 17 \mathrm{GV}\right)$. While a comparison of count rates with those at other monitors could only provide spectral information for $P_{c}<17$ $\mathrm{GV}$, use of time-delay information to infer the leader fraction (a proxy for the spectral index) provides information about rigidities above $17 \mathrm{GV}$, thus extending the reach of the worldwide NM network to higher rigidity.

\subsection{Charge Sign Dependence (4)}

As noted earlier, the effects of solar magnetic polarity on cosmic ray transport depend on the product $q A$, so these effects can be explored by simultaneous flux measurements of cosmic rays of opposite charge sign. In this conference, data on the positron fraction, $e^{+} /\left(e^{+}+e^{-}\right)$, were presented by the PAMELA collaboration for energies of 0.5-5 GeV during 2006-2015 [62]. This ratio jumped upward in 2015 (especially at $E<2.5 \mathrm{GeV}$ ) after the solar polarity reversal of 2012- 
2014, which confirms a pattern of charge sign dependence observed during previous solar cycles (e.g., [22]), including results presented here for Solar Cycle 23 [59].

[78] stated that the record high cosmic ray proton flux in 2009 (near the time of sunspot minimum) was unexpected because that corresponded to $q A<0$. The motivation for this statement can be seen from Figure 14. Thus they predict that the upcoming GCR ion peak could reach an even higher flux!

Figure 14: Observations of the GCR proton spectrum near times of solar minimum (GCR maximum) for various solar cycles with $A>0$ (blue) and $A<0$ (red). Usually $A<0$ conditions led to lower proton fluxes, especially below $0.3 \mathrm{GeV}$, but during the record-high GCR maximum of 2009 the spectrum was much higher, despite the $A<0$ conditions. This leads the authors to predict an even higher GCR maximum near the end of the present decade (from presentation by [78]).

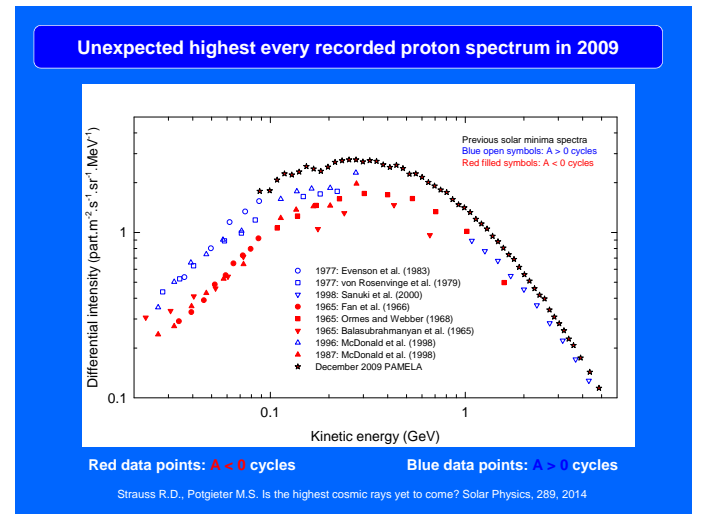

\subsection{Outer Heliosphere (3)}

It is exciting that the two Voyager spacecraft continue to provide data as they explore the outer heliosphere and space beyond the heliopause. [89] presented $\mathrm{H}, \mathrm{He}$, and $\mathrm{O}$ energy spectra with features corresponding to termination shock particles (TSPs), anomalous cosmic rays (ACRs), and GCRs. At the time when the Voyager spacecraft passed near the nose of the solar wind termination shock, counter to expectations it was found that higher-energy ACRs were not accelerated locally in the shock nose region. From their analysis, they conclude that $0.5-35 \mathrm{MeV}$ protons came from the flank of the termination shock, as suggested by [52], and $28-43 \mathrm{keV}$ protons are convected with the solar wind from the nose of the termination shock.

Other presentations concerned how to understand GCR variations observed by Voyager-1 beyond the heliopause [39] and the acceleration of Galactic electrons at the solar wind termination shock in relation to Voyager-1 observations [77].

\subsection{Modeling (6)}

There were several presentations about the very challenging effort to model GCR transport throughout the entire heliosphere and match observations of time-dependent solar modulation. The effects of the solar magnetic field and the heliospheric current sheet were studied by [80, 81]. Cosmic ray transport in the heliosphere was studied using the HELIOPROP calculation framework [97]. Drift effects in the 22-year cycle of GCR modulation were modeled by [40]. The combined modulation of Jovian and Galactic electrons in the heliosphere was examined by [76]. Finally, a simplified ab initio model in an effort to avoid any empirical model parameters was presented by [61]. 


\subsection{Anisotropy (3)}

Furthermore, the solar diurnal GCR anisotropy can be studied using ground-based detectors by examining the daily variation in count rates with Earth's rotation. [72] examined the solar cycle dependence of the diurnal variation measured by the SMS muon detector. Long-term variations of vector and tensor anisotropies of cosmic rays were analyzed by [28]. There were also modeling results for GCR anisotropy at the high-energy end of solar modulation [38].

\subsection{Forbush Decreases (10)}

The final topic of this Rapporteur Paper is Forbush decreases (FDs), which involve temporary decreases in the GCR flux due to solar storms or high speed solar wind streams. There was a survey of the properties of FDs observed by the Alpha Magnetic Spectrometer on the International Space Station [69]. Cosmic ray intensity variations associated with corotating interaction regions (related to high speed solar wind streams) were studied using data from PAMELA [63].

The GRAPES-3 experiment in Ooty, India reported that during an FD, on 2015 June 22 the GCR flux rose rapidly approximately to the pre-decrease level and then declined again after $\approx 1$ hour [60]. These rapid changes were interpreted in terms of a temporary decrease in the geomagnetic cutoff. Another report considered both that event and an impulsive increase in the GCR flux on 2017 January 18 as observed by IceTop, the surface array component of the IceCube Neutrino Observatory at the South Pole [33]. A GCR flux increase at the South Pole cannot be attributed to decrease in the geomagnetic cutoff. For the event of 2015 June 22, these authors point out that any change in geomagnetic cutoff should be global, but the worldwide NM network recorded flux increases only at certain longitudes, so the flux variation during that FD seems more consistent with an anisotropy effect. That FD was also studied with the LAGO detector in Brazil [20].

A theory of an FD in a magnetic cloud was presented by [71]. FDs were measured by the scintillation muon hodoscope ( $\mathrm{ScMH}$ ) [104]. Cosmic ray variations were measured at the Carpet/ENU detector at Astana in October-November, 2016 [26]. There was an investigation of short-term variations of vector and tensor anisotropies using a magnetic mirror model [29]. Finally, there was a presentation of geomagnetic cutoff calculations for the interpretation of low-rigidity cosmic-ray antiparticle measurements [98].

\section{Acknowledgments}

We thank the many participants in the Solar-Heliospheric (SH) sessions at the 35th International Cosmic Ray Conference for their presentations and for kindly explaining their work. It is a privilege to summarize their work in the present manuscript.

\section{References}

[1] Aleksandrin, S., Galper, A., Koldashov, S., Mikhailov, V., \& Zharaspayev, T., these proceedings

[2] Amenomori, M., Bi, X., Chen, D., Chen, T., Chen, W.,et al., these proceedings

[3] Amenomori, M., Bi, X., Chen, D., Chen, W., Cui, S., et al., these proceedings

[4] Ammosova, A., Gavrilyeva, G., Ammosov, P., \& Koltovskoi, I., these proceedings 
[5] Ammosova, A., Gavrilyeva, G., Ammosov, P., \& Koltovskoi, I., these proceedings

[6] Anzorena, M., Valdés-Galicia, J.F., Garcia, R., Matsubara, Y., Sasai, Y., et al., these proceedings

[7] Anzorena, M., Valdés-Galicia, J.F., Garcia, R., Matsubara, Y., Sasai, Y., et al., these proceedings

[8] Arunbabu, K.P., Dugad, S.R., K.P., Gupta, S.K., Hariharan, B., Hayashi, Y., et al., these proceedings

[9] Banglieng, C., Ruffolo, D., Sáiz, A., Evenson, P., \& Nutaro, T., these proceedings

[10] Bashindzhagyan, G., Barnes, V., Fischbach, E., Hovsepyan, G., Korotkova, N., et al., these proceedings

[11] Braga, C.R., Mendonça, R., Echer, E., Lago, A.D., Pinto, A., et al., these proceedings

[12] Bramlitt, E., these proceedings

[13] Bramlitt, E., these proceedings

[14] Burger, R.A., \& Engelbrecht, N.E., these proceedings

[15] Chen, J., \& Bieber, J. W. 1993, ApJ, 405, 375

[16] Chen, S., Nan, Y., On behalf of the ARGO-YBJ Collaboration, these proceedings

[17] Cohen, C., Luhmann, J.G., Mewaldt, R.A., Mays, M.L., Bain, H.M., et al., these proceedings

[18] Desai, M., these proceedings

[19] Engelbrecht, N.E., Strauss, R.D., leRoux, J.A., \& Burger, R.A., these proceedings

[20] Fauth, A., Souza, H., Boezio, and the LAGO Collaboration, these proceedings

[21] García, R., Valdés-Galicia, J.F., Anzorena, M., Ortiz, E., González, L.X., et al., these proceedings

[22] Garcia-Munoz, M., Meyer, P., Pyle, K. R., Simpson, J. A., \& Evenson, P. 1986, JGR, 91, 2858

[23] Gil, A., \& Alania, M.V., these proceedings

[24] Gil, A., Asvestari, E., Kovaltsov, G.A., \& Usoskin, I., these proceedings

[25] Gil, A., \& Mursula, K., these proceedings

[26] Giniyatova, S., these proceedings

[27] Gololobov, P.Yu., Grigoryev, V.G., \& Starodubtsev, S.A., these proceedings

[28] Gololobov, P.Yu., Krymsky, G.F., \& Krivoshapkin, P.A., these proceedings

[29] Gololobov, P.Yu., Krymsky, G.F., \& Krivoshapkin, P.A., these proceedings

[30] González, L.X., Valdés-Galicia, J.F., Muraki, Y., Watababe, K., Sako, T., et al., these proceedings

[31] Ho, G., these proceedings

[32] IceCube Collaboration, Mangeard, P.S., Muangha, P., Pyle, R., Ruffolo, D., \& Sáiz, A., these proceedings

[33] IceCube Collaboration, Mangeard, P.S., Muangha, P., Pyle, R., Ruffolo, D., \& Sáiz, A., these proceedings

[34] Jokipii, J. R., \& Thomas, B. 1981, ApJ, 243, 1115

[35] Kamiya, K., Kogo, K., Masuda, S., Matsumoto, H., Muraki, Y., et al., these proceedings

[36] Kamiya, K., Kogo, K., Masuda, S., Matsumoto, H., Muraki, Y., et al., these proceedings 
[37] Kocharov, L., Mishev, A., Pohjolinen, S., Reiner, M., Lee, J., et al., these proceedings

[38] Kóta, J., Kozai, M., \& Munakata, K., these proceedings

[39] Kóta, J., these proceedings

[40] Kóta, J., these proceedings

[41] Krüger, H., these proceedings

[42] Krüger, H., these proceedings

[43] Krüger, P.P., Krg̈er, H.G., Krüger, H., Diedericks, C., \& Malan, D., these proceedings

[44] Labrador, A., Sollitt, L., Cohen, C., Cummings, A., Leske, A., et al., these proceedings

[45] Lara, A., Caballero-Lopez, R.A., these proceedings

[46] Lara, A., Raga, G.B., Enríquez-Rivera, O., for the HAWC collaboration, these proceedings

[47] Le, G., \& Zhang, X., these proceedings

[48] Leske, R., Cummings, A., Cohen, C., Mewaldt, R., Labrador, A., et al., these proceedings

[49] Lidvansky, A., these proceedings

[50] Mangeard, P.S., Clem, J., Evenson, P., Pyle, R., Mitthumsiri, W., et al., these proceedings

[51] Mattana, V., these proceedings

[52] McComas, D. J., \& Schwadron, N. A. 2006, GeoRL, 33, 4102

[53] Mergè, M., Bruno, A., Martucci, M., Adriani, O., Barbarino, G., et al., these proceedings

[54] Mewaldt, R., Li, G., Hu, J., \& Cohen, C., these proceedings

[55] Mishev, A., Artamonov, A., Kovalstov, G., Mironova, I., \& Usoskin, I., these proceedings

[56] Mishev, A., Artamonov, A., Kovalstov, G., \& Usoskin, I., these proceedings

[57] Mishev, A., Usoskin, I., \& Kocharov, L., these proceedings

[58] Mishev, A. \& Velinov, P., these proceedings

[59] Miyake, S., Yanagita, S., these proceedings

[60] Mohanty, P.K., Arunbabu, K.P., Dugad, S.R., Gupta, S.K., Hariharan, B., et al., these proceedings

[61] Moloto, K.D., these proceedings

[62] Munini, R., Felice, V., Boezio, M., Adriani, O., Barbarino, G.C., et al., these proceedings

[63] Munini, R., Felice, V., Boezio, M., Adriani, O., Barbarino, G.C., et al., these proceedings

[64] Muraki, Y., these proceedings

[65] Muraki, Y., Valdés-Galicia, J., González, L., Kamiya, K., Katayose, Y., et al., these proceedings

[66] Otsuka, F., Matsukiyo, S., \& Hada, T., these proceedings

[67] Park, J., these proceedings

[68] Park, J., these proceedings

[69] Palermo, M., these proceedings

[70] Petukhova, A., Petukhov, I., \& Petukhov, S., these proceedings 
[71] Petukhova, A., Petukhov, I., \& Petukhov, S., these proceedings

[72] Pinto, A.C.S., Mendonca, R.R.S., Braga, C.R., Dal Lago, A., Echer, E., \& Schuch, N.J., these proceedings

[73] Poluianov, S., Kovaltsov, G., Mishev, A.,\& Usoskin, I., these proceedings

[74] Poluianov, S., Kovaltsov, G. \& Usoskin, I., these proceedings

[75] Poluianov, S., Usoskin, I., Mishev, A., Smart, D., Shea, M., these proceedings

[76] Potgieter, M., \& Nndanganeni, R., these proceedings

[77] Potgieter, M., Prinsloo, P.L., \& Strauss, R.d.T., these proceedings

[78] Potgieter, M., Vos, E., Bisschoff, D., Raath, J.L., Boezio, M., et al., these proceedings

[79] Protopopov, G., Anashin, V., Lyakhov, I., Rukavichnikov, S., Denisova, V., \& Tsurgaev, A., these proceedings

[80] Raath, J.L., these proceedings

[81] Raath, J.L., these proceedings

[82] Raath, J.L., \& Potgieter, M.S., these proceedings

[83] Rajiv, K., these proceedings

[84] Ruffolo, D., Sáiz, A., Mangeard, P.-S., et al. 2016, ApJ, 817, 38

[85] Ruffolo, D., Tooprakai, P., Rujiwarodom, M., et al. 2006, ApJ, 639, 1186

[86] Sáiz, A., Mitthumsiri, W., Ruffolo, D., Evenson, P., \& Nutaro., T, these proceedings

[87] Sasai, Y., Matsubara, Y., Itow, Y., Sako, T., Kawabata, T., et al., these proceedings

[88] Stenkin, Y., Alekseenko, V., Cui, S., He, Y., Li, B., et al., these proceedings

[89] Stone, E., Cummings, A., Heikkila, B., Lal, N., \& Webber, W., these proceedings

[90] Strauss, R.D., Dressing, N., \& Engelbrecht, N.E., these proceedings

[91] Suzuki, S., Sakurai, H., Tokanai, F., Inui, E., Shimizu, H., et al., these proceedings

[92] Timofeev, V., Timofeev, L., \& Samsonov, S., these proceedings

[93] Tooprakai, P., Seripienlert, A., Ruffolo, D., Chuychai, P., \& Matthaeus, W.H., these proceedings

[94] Usoskin, I., these proceedings

[95] Usoskin, I., Asvestari, E., Willamo, T., Gil, A., Kovaltsov, G., et al., these proceedings

[96] Velinov, P., Balabin, Y., \& Maurchev, E., these proceedings

[97] Vittino, A., Evoli, C., \& Gaggero, D., these proceedings

[98] von Doetinchem, P., \& Yamashiro, B., these proceedings

[99] Wang, X., Yan, Y., Ding, M., Wang, N. \& Shan, H., these proceedings

[100] Wang, X., Yan, Y., Ding, M., Wang, N. \& Shan, H., these proceedings

[101] Watanabe, K., Sasuda, S., \& Ohno, M., these proceedings

[102] Wawrzynczak, A., these proceedings

[103] Wiedenbeck, M., these proceedings

[104] Yashin, I., Ampilogov, N.V., Astapov, I., Barbashina, N., Dmitrieva, A., et al., these proceedings

[105] Yu, X.X., Jin, C.L., \& An, Z.H., these proceedings 